\title{
A New Lunar DEM Based on the Calibrated Chang'E-1 Laser Altimeter Data
}

\author{
Yong Huang $\mathbb{D}^{1,2}$ Shengqi Chang, ${ }^{1,3}$ Songhe Qin, ${ }^{1,2}$ Peijia Li $(\mathbb{D}){ }^{1}$ \\ Xiaogong Hu, ${ }^{1}$ and Min Fan $^{4}$ \\ ${ }^{1}$ Key Laboratory of Planetary Sciences, Shanghai Astronomical Observatory, Chinese Academy of Sciences, Shanghai 200030, China \\ ${ }^{2}$ University of Chinese Academy of Sciences, Beijing 100049, China \\ ${ }^{3}$ Qianxun Spatial Intelligence Inc., Shanghai 200438, China \\ ${ }^{4}$ Key Laboratory of Space Object Measurement, Beijing 100094, China
}

Correspondence should be addressed to Yong Huang; yongh@shao.ac.cn

Received 2 April 2018; Accepted 20 May 2018; Published 19 June 2018

Academic Editor: Jianguo Yan

Copyright (C) 2018 Yong Huang et al. This is an open access article distributed under the Creative Commons Attribution License, which permits unrestricted use, distribution, and reproduction in any medium, provided the original work is properly cited.

To improve the lunar DEM accuracy derived from CE-1 altimeter data, CE-1 laser altimeter data are calibrated in this paper. Orbit accuracy and ranging accuracy are the two most important factors to affect the application of altimeter data in the lunar topography. An empirical method is proposed to calibrate CE-1 altimeter data, using gridded LOLA DEM to correct systematic errors of CE-1 altimeter data, and the systematic bias is about $-139.52 \mathrm{~m}$. A new lunar DEM grid model based on calibrated CE-1 altimeter data with the spatial resolution of $0.0625^{\circ} \times 0.0625^{\circ}$ is obtained as well as a spherical harmonic model at 1400th order. Furthermore, the DEM accuracy is assessed through the comparison with the nearside landmarks of the Moon, and the results show that the DEM accuracy is improved from $127.3 \mathrm{~m}$ to $48.7 \mathrm{~m}$ after the calibration of laser altimeter data.

\section{Introduction}

Lunar topography plays an important role in lunar exploration [1]. Benefiting from several lunar explorers launched in recent 20 years, humans have reached a high level to understand the lunar topography. After Clementine was launched in 1994, Japan, China, and India launched lunar explorers SELENE (Selenological and Engineering Explorer), CE-1 (ChangE-1), and Chandrayaan-1, respectively, between the year of 2007 and 2008. In 2009 the US launched LRO (Lunar Reconnaissance Orbiter), which provided accurate laser altimeter data and CCD image data for scientists to study the lunar topography [2-7].

With the increasing topography measurements, the study of lunar topography no longer depends on one single probe or one single type of data. Data from different devices of the same probe can be used to verify each other and improve the data quality. For example, the combined adjustment of CE-1 laser altimeter data and CCD image data can improve the spatial resolution of a topography model effectively $[8,9]$. Barker solved lunar topography by combining data from LRO Lunar
Orbiter Laser Altimeter (LOLA) and TC (Terrain Camera) deployed on SELENE's main satellite Kaguya, and the results showed that TC data can make up for gaps in the spatial distribution of LOLA and improve the resolution of the topography model [10].

LAM (laser altimeter) deployed on CE-1 obtained about total 9.12 million measurements covering the whole lunar surface. Ping et al. developed a lunar global digital elevation model (DEM) CLTM-s01 with 360th order based on the 3 million effective laser altimetry observations acquired during the first official flight of CE-1. The space resolution was better than $0.5^{\circ}$, and the elevation accuracy of the model was about $31 \mathrm{~m}$ [11]. Li et al. developed a DEM with $3 \mathrm{~km}$ resolution. The model had a planar positioning accuracy of $445 \mathrm{~m}$ and an elevation accuracy of $60 \mathrm{~m}$ [12]. Cai et al. proposed a hierarchical many-knot spline method and developed a global lunar DEM with a $0.0625^{\circ}$ resolution based on more than 8.2 million data obtained from CE-1 [13]. Hu reprocessed LAM data by using the crossover adjustment method. The lunar surface was divided into 32 regions for the least square adjustment, 
TABLE 1: Specifications of altimetry instrument on CE-1/SELENE/LRO.

\begin{tabular}{lccc}
\hline & CE-1 & SELENE & LRO \\
\hline Orbit altitude & $200 \mathrm{~km}$ & $100 \mathrm{~km}$ & $50 \mathrm{~km}$ \\
Instrument & Laser AltiMeter & Laser ALTtimeter & $4.1 \mathrm{~m}$ \\
Ranging error & $5 \mathrm{~m}$ & $1 \mathrm{~Hz}$ & $<1 \mathrm{~m}$ \\
Sampling rate & $1 \mathrm{~Hz}$ & $1 \mathrm{~m}$ & $28 \mathrm{~Hz}$ \\
Radial orbit error & $15 \mathrm{~m}$ & $\sim 50 \mathrm{~m}$ & $1 \mathrm{~m}$ \\
3D orbit error & $\sim 200 \mathrm{~m}$ & $\sim 40 \mathrm{~m}$ & $\sim 20 \mathrm{~m}$ \\
Footprint & $<200 \mathrm{~m}$ & $5 \mathrm{~m} / \mathrm{beam}$ & \\
\hline
\end{tabular}

and the newly obtained topography model was improved significantly on the regional scale [14].

The orbit accuracy and ranging accuracy are the two most important factors to affect the application of altimeter data in the lunar topography. The altimeter measurements include systematic errors and random errors, and the systematic errors should be calibrated carefully before the lunar topography calculation. The mean radius of the Moon derived from Chang'E-1 is $1737013 \pm 2 \mathrm{~m}$ [11], which has an obvious bias of nearly $140 \mathrm{~m}$ when compared with those derived from LRO (1737153 $\pm 10 \mathrm{~m})$ and LALT $(1737150 \pm 10 \mathrm{~m})[2,15]$, and indicates that CE-1 LAM data has a systematic bias and should be removed before DEM calculation. Ping et al. also estimated the possible systematic bias of reference frequency in CE-1 LAM oscillator, and the result is about $145 \mathrm{~m} \mathrm{[16].} \mathrm{In} \mathrm{this}$ paper, a different method was used to calibrate CE-1 LAM data systematic errors, using LOLA data as the benchmark to calibrate and remove systematic errors of the data from CE-1 LAM data, and a new lunar DEM was generated using calibrated LAM data.

\section{Orbit Improvement and Calibration of LAM Data}

CE-1 was tracked by Unified S-Band (USB) ranging and Very Long Baseline Interferometry (VLBI) technique. To improve the orbit accuracy of CE-1, the altimeter crossover data were used in the orbit determination. We reprocessed the CE-1 orbit by combining the ground tracking data as well as the altimetry crossover data, and the orbit accuracy was increased from $147 \mathrm{~m}$ to $105 \mathrm{~m}$, which was about $30 \%$ improvement compared with the result using ground tracking data only [17].

It is important to eliminate altimeter data errors by crosscorrecting. The altimeter data contain systematic errors consisted of orbit errors, positioning errors of laser footprints on lunar surface, time tag, and instrument errors. In this paper, an empirical data calibration method was used to remove the systematic errors remaining in LAM data.

As Table 1 shows, LOLA ranging error was less than $1 \mathrm{~m}$, which was better than LAM's $5 \mathrm{~m}$ accuracy and LALT's $4 \mathrm{~m}$ accuracy, and the orbit accuracy of LRO was also much better than that of CE-1 and SELENE. Therefore, the LOLA data were used as the benchmark to calibrate possible systematic errors in LAM data before the DEM calculation.
In this paper, the CE-1 LAM observations, namely, LAM 2B-SHAO data, were about 7 million, in about 5 months (2007.11.28-2008.02.06 and 2008.05.15-2008.07.07), which were obtained based on the improved orbit [17]. The LOLA data used here were about 1 year during the LRO mapping orbit from 2009.9.26 to 2010.8.20 [15], which were the initial observations from LRO, and LOLA data acquisition continued up to 2015 [18].

The LOLA data were firstly used to develop a reliable topography model, which would be used to calibrate LAM data. The following model was used to calculate systematic errors in LAM data.

$$
\begin{aligned}
\Delta h(t)= & h(t)-h_{M} \\
= & b+\dot{h} \tau \\
& +\sum_{k=1}^{\ell}\left[S_{k} \sin \omega_{k}\left(t-t_{0}\right)+C_{k} \cos \omega_{k}\left(t-t_{0}\right)\right]
\end{aligned}
$$

In formula (1), $h(t)$ was the elevation derived from LAM data at the time $t . h_{M}$ was the elevation of the same point as $h(t)$ calculated from the LOLA topography model. And $\Delta h(t)$ was the elevation residual. $\dot{h}$ was the rate of height change. We modeled the elevation residual $\Delta h(t): b$ was the constant bias, $\tau$ was the time tag deviation, $\omega_{k}$ was the frequency, $S_{k}$ and $C_{k}$ were used to characterize the error relating to the orbit, and $t_{0}$ was the initial epoch time. $b, \tau, S_{k}$, and $C_{k}$ were the parameters to be estimated using the least square method. The value of $\omega_{k}$ could be obtained by doing Fourier transform on $\Delta h(t)$.

Figure 1 showed the time series of the residual $\Delta h(t)$ of LAM data. In the calculation of $h_{M}$, nearest neighbor interpolation was used. In order to determine the characteristic frequency, we needed to carry out Fourier spectrum analysis on $\Delta h(t)$, and the results were shown in Figure 2. Judging from the spectral analysis in Figure 2, the main periodic terms in the elevation residuals were $\sim 129$ min (about 1 orbit period) for LAM data. Because the orbit error was dominated by the error at a frequency of once per orbital revolution (1 cpr), only 1 cpr orbital error was estimated (i.e., $l=1$ in (1)).

The calibration results were shown in Table 2 . For comparison, the calibration result of SELENE LALT data was also shown in Table 2. The result indicated that errors in LALT data were smaller than those in LAM data. The constant bias of LAM was $-139.52 \mathrm{~m}$, which was the same level as Ping's analysis of the overall deviation of ground crystal oscillator [16]. The time tag deviations in LAM and LALT were very small. 
The orbital error based on LAM data was about $5 \mathrm{~m}$, and the orbital error based on LALT data was less than $1 \mathrm{~m}$, both of which were smaller than the real radial orbit errors of CE-1 and SELENE, respectively, indicating that the method cannot remove orbital errors totally. We used the above estimated results to correct LAM data, and the corrected data were adopted in the DEM calculation. For the sake of clarity, the modified LAM 2B-SHAO data were named as LAM 2B-SHAO-cali.

\section{The Lunar DEM Based on the Calibrated CE-1 LAM Data}

After LAM data systematic errors correction, we developed a lunar DEM based on the calibrated CE-1 altimeter data with a $0.0625^{\circ}$ resolution. In this paper, we used a quadratic fitting method to interpolate grid points. The formulae were as follows:

$$
\begin{aligned}
& h\left(\lambda_{i}, \varphi_{i}\right)=\mathrm{A}_{\mathrm{i}} \cdot \xi
\end{aligned}
$$

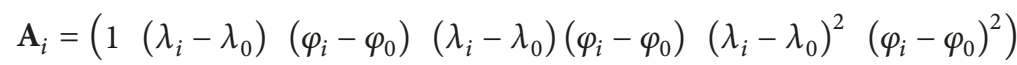

$$
\begin{aligned}
& \xi=\left(\begin{array}{llllll}
a_{0} & a_{1} & a_{2} & a_{3} & a_{4} & a_{5}
\end{array}\right)^{T}
\end{aligned}
$$

where $\left(\lambda_{i}, \varphi_{i}\right)$ and $h$ were the lunar longitudes, latitudes, and elevation of the laser altimetry footprints in the grid, respectively. $\left(\lambda_{0}, \varphi_{0}\right)$ were the longitude and latitude of the grid point to be interpolated, and $a_{0} \sim a_{5}$ were 6 parameters to be estimated. The fitting result $a_{0}$ was the elevation value of the grid points to be interpolated.

Because CE-1 was a polar-orbiting satellite, the altimeter data were mainly distributed along the longitude. In order to avoid the abnormal solution as a result of few or poor distribution of the altimeter data, when the correlation coefficient of the fitting result was too large (greater than 105) or the measurement in the grid was less than 50, we applied the inverse distance weighted interpolation method whose distance weight function followed Gaussian attenuation to calculate the elevation of the grid point:

$$
\begin{aligned}
\widehat{h} & =\frac{\sum_{j=1}^{n} w_{j} h_{j}}{\sum_{j=1}^{n} w_{j}} \\
w_{j} & =\exp \left(-\left(\frac{d_{j}}{D}\right)^{2}\right) \cos \varphi_{j}
\end{aligned}
$$

where $d_{j}$ was the distance of $h_{j}$ to the grid center. $D$ was the value of grid width at $0.0625^{\circ}$ in this paper.

Figure 3 showed the results of the DEM, in which the elevation value referred to the mean lunar radius of 1737.4 $\mathrm{km}$. Figure 3 offered a global Mollweide projection, and the central longitude was $90^{\circ} \mathrm{W}$.

Three topography models based on the original LAM data, LAM data with orbit improvement, and LAM data with calibration, respectively, were compared to the topography model derived from LOLA data. Figure 4 showed the differences. In Figure 4(a), stripped differences could be seen clearly, which were mainly caused by the orbit error. With the new orbit results, no more significant stripped differences could be found in Figure 4(b). There was still an obvious bias in Figures 4(a) and 4(b) from the color bar, which was weakened a lot in Figure 4(c) based on the calibrated LAM data after orbit improvement.
Table 3 exhibited the highest and lowest points of lunar topography obtained from different DEMs. Among them, the result of CE-1 (Li Chunlai) was obtained by Li Chunlai et al. using all of about 9.12 million altimeter data [12]. The result of LOLA was obtained by Smith using LOLA measurements over seven years up to 2015 [18]. The positions and elevations of the highest points in all models were almost the same, which were located on the uplifts adjacent to the Korolev and Dirichlet-Jackson basins on the far side of the Moon. The lowest point of DEM obtained using calibrated LAM data in this paper was quite different from the other two models. According to Li Chunlai's analysis, the lowest point from LAM data was obtained at Beijing time 2008.07.15-13: 52: 29.778, but the LAM data used in this paper ended in 2008-07-07 for the limit of precise orbit. Therefore, the position and elevation of the lowest point in the DEM obtained by LAM-2B-SHAOcali data were quite different from those in other DEMs. This result was similar to the CLTM-s01 model obtained by the first 3 months of CE-1 LAM data [11].

The grid model of topography could be expanded to a spherical harmonic form according to

$$
\begin{aligned}
& H(\lambda, \varphi) \\
& =\sum_{l=0}^{N} \sum_{m=0}^{l} \bar{P}_{l m}(\sin \varphi)\left(\bar{C}_{l m} \cos (m \lambda)+\bar{S}_{l m} \sin (m \lambda)\right) \\
& \bar{C}_{l m} \\
& =\frac{\Delta \lambda \Delta \theta}{4 \pi} \sum_{i=1}^{A} \sum_{j=1}^{B} H\left(\lambda_{i}, \varphi_{j}\right) \bar{P}_{l m}\left(\sin \varphi_{j}\right) \cos \left(m \lambda_{i}\right) \cos \varphi_{i} \\
& \bar{S}_{l m} \\
& =\frac{\Delta \lambda \Delta \theta}{4 \pi} \sum_{i=1}^{A} \sum_{j=1}^{B} H\left(\lambda_{i}, \varphi_{j}\right) \bar{P}_{l m}\left(\sin \varphi_{j}\right) \sin \left(m \lambda_{i}\right) \cos \varphi_{i}
\end{aligned}
$$

In formula (7), $\lambda$ and $\varphi$ referred to the longitude and latitude, respectively, $\bar{P}_{l m}$ referred to the normalized Legendre function, $\bar{C}_{l m}$ and $\bar{S}_{l m}$ referred to the normalized spherical 


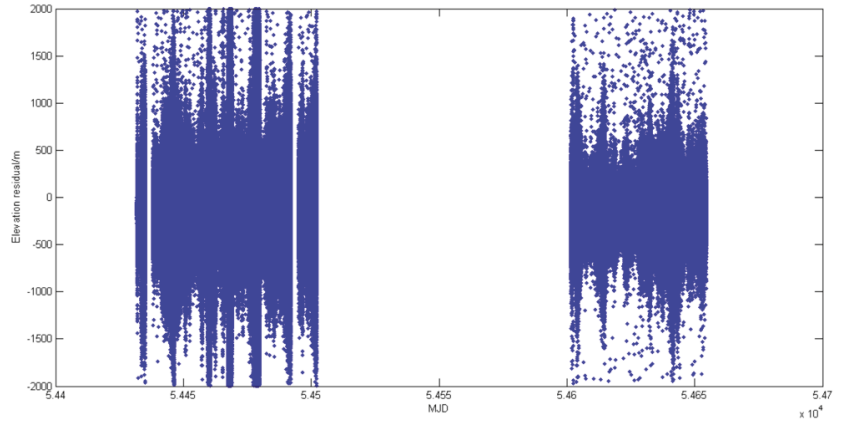

FIGURE 1: Residuals of LAM data compared with LOLA topography model.

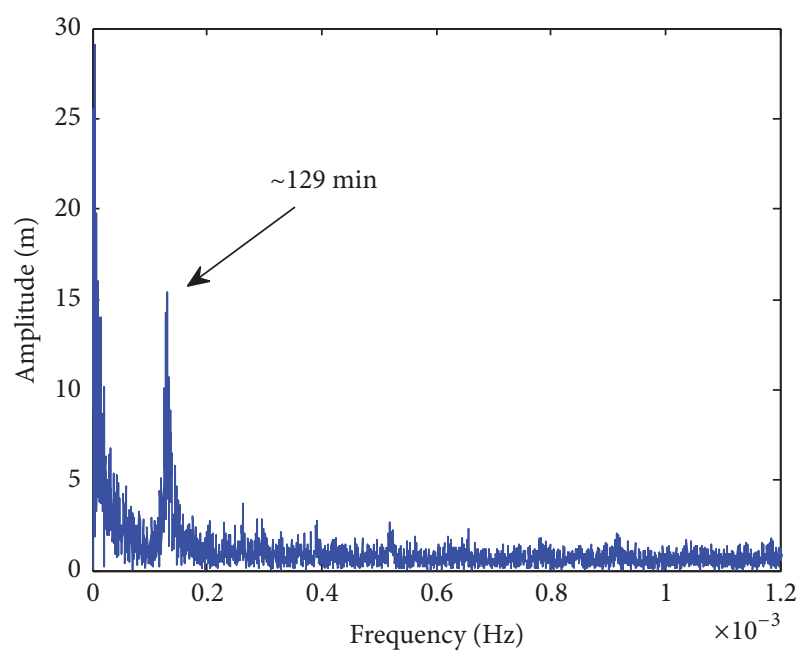

FIGURE 2: Spectral analysis of LAM residuals.

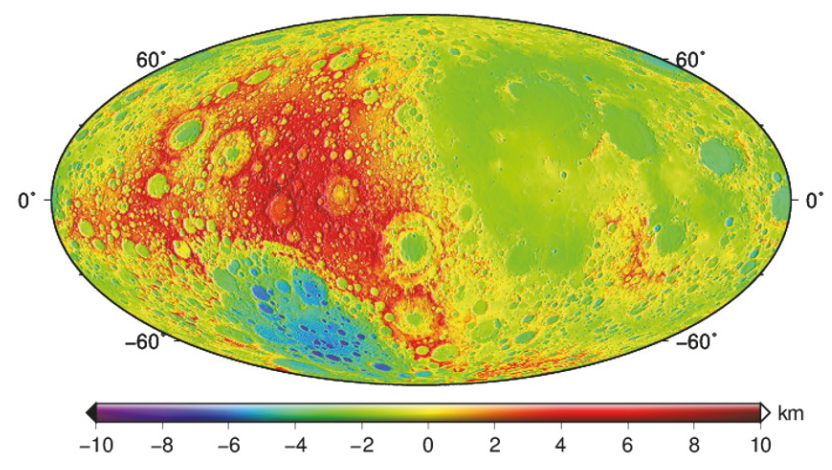

FIGURE 3: Lunar topography model based on the calibrated LAM data.

harmonic coefficients, the summation index $N$ referred to the max order, and $A$ and $B$ referred to the number of grid points in the latitudinal and longitudinal directions, respectively. We expanded the spherical harmonic coefficients to the 1400th order according to the above formula.

Based on the results of spherical harmonic expansion, the mean radius of the Moon was $1737.152 \mathrm{~km}$, the polar radius was $1735.658 \mathrm{~km}$, the equatorial radius was $1737.613 \mathrm{~km}$,

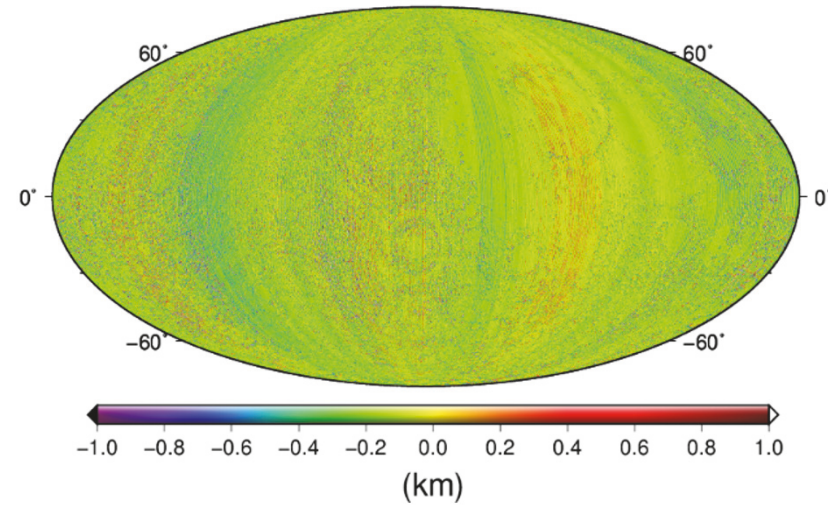

(a) Original LAM data

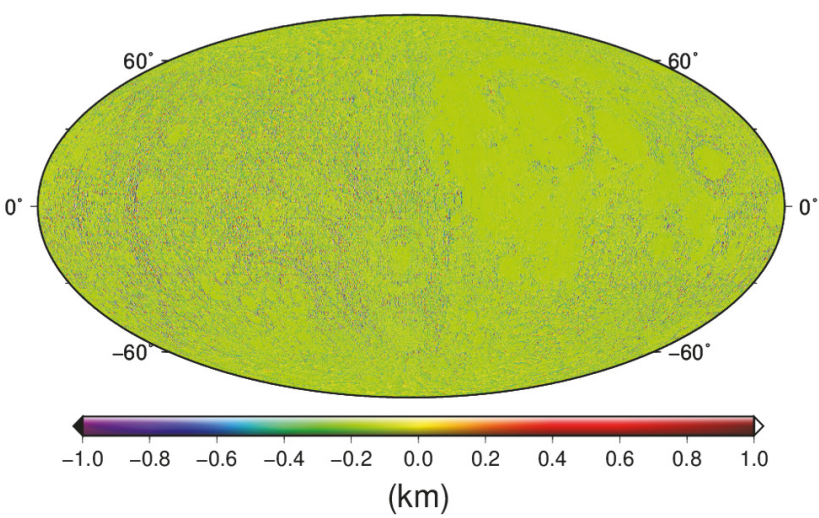

(b) LAM data with orbit improvement

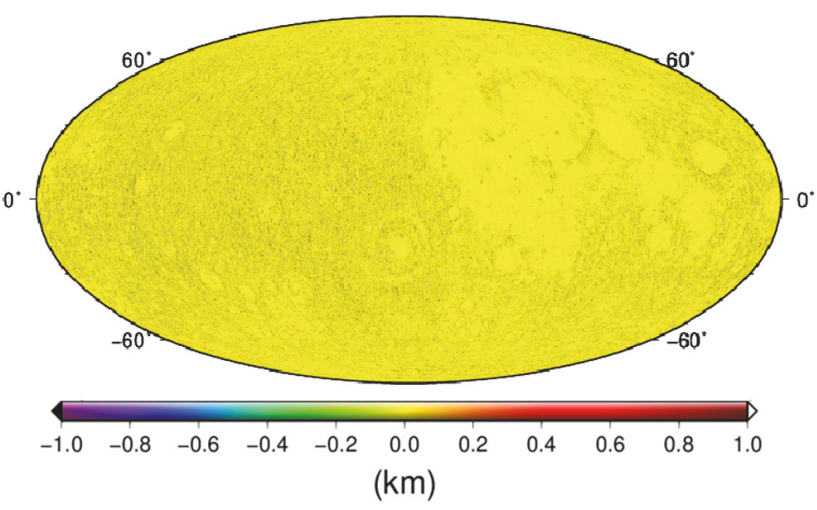

(c) LAM data with calibration

FIGURE 4: Differences of DEMs compared to LOLA DEM. (a) model from original LAM data, (b) model from LAM data with orbit improvement, and (c) model from LAM data with calibration.

TABLE 2: Results of LAM and LALT data calibration.

\begin{tabular}{lcccc}
\hline & $b / \mathrm{m}$ & $\tau / \mathrm{s}$ & $S_{k} / \mathrm{m}$ & $C_{k} / \mathrm{m}$ \\
\hline LAM & -139.52 & -0.0073 & 5.25 & -4.21 \\
LALT & 3.03 & -0.0780 & 0.26 & -0.24 \\
\hline
\end{tabular}

and the lunar flattening rate $1 / \mathrm{f}$ was $1 / 887.70$. The lunar COF (Center of Figure) and COM (Center of Mass) had a deviation of $0.241 \mathrm{~km}$ from the north of the lunar rotation axis, and 
TABLE 3: The highest and lowest points from different lunar DEMs.

\begin{tabular}{lccccccc}
\hline Data source & \multicolumn{3}{c}{ Highest point of lunar surface } & \multicolumn{2}{c}{ Lowest point of lunar surface } & \multicolumn{2}{c}{ Max elevation } \\
& Longitude & Latitude & Elevation $/ \mathrm{km}$ & Longitude & Latitude & Elevation $/ \mathrm{km}$ & difference $/ \mathrm{km}$ \\
\hline CE-1 (Li Chunlai) & $158.656^{\circ} \mathrm{W}$ & $5.441^{\circ} \mathrm{N}$ & 10.629 & $172.413^{\circ} \mathrm{W}$ & $70.368^{\circ} \mathrm{S}$ & -9.178 & 19.807 \\
LAM-2B-SHAO -cali & $158.594^{\circ} \mathrm{W}$ & $5.469^{\circ} \mathrm{N}$ & 10.723 & $148.625^{\circ} \mathrm{W}$ & $61.375^{\circ} \mathrm{S}$ & -8.531 & 19.254 \\
LOLA & $158.63^{\circ} \mathrm{W}$ & $5.341^{\circ} \mathrm{N}$ & 10.792 & $172.48^{\circ} \mathrm{W}$ & $70.36^{\circ} \mathrm{S}$ & -9.129 & 19.921 \\
\hline
\end{tabular}

TABLE 4: LLRR and ALSEP elevation error for different DEMs.

\begin{tabular}{|c|c|c|c|c|}
\hline Reflector & Original LAM & LAM-2B-SHAO & LAM-2B-SHAO_cali & LOLA (initial phase) \\
\hline Lunakhod 1 (LRRR) & -146.14 & -141.66 & -3.27 & -3.66 \\
\hline Lunakhod 2 (LRRR) & -11.40 & -48.37 & 65.62 & -17.19 \\
\hline Apollo 11 (LRRR) & -140.17 & -99.99 & 87.75 & -0.4 \\
\hline Apollo 14 (LRRR) & -145.59 & -85.45 & -1.04 & 6.3 \\
\hline Apollo 15 (LRRR) & -180.92 & -185.89 & -49.72 & -20.18 \\
\hline Apollo 12 (ALSEP) & -158.49 & -83.7 & -14.52 & -23.15 \\
\hline Apollo 14 (ALSEP) & -160.59 & -100.45 & -16.04 & -8.7 \\
\hline Apollo 15 (ALSEP) & -180.92 & -185.89 & -49.72 & -20.18 \\
\hline Apollo 16 (ALSEP) & -187.24 & -184.35 & -16.72 & -36.29 \\
\hline Apollo 17 (ALSEP) & -66.29 & -46.61 & 77.48 & -11.81 \\
\hline $\mathrm{RMS} / \mathrm{m}$ & 147.70 & 127.31 & 48.70 & 17.98 \\
\hline
\end{tabular}

a deviation of $-1.782 \mathrm{~km}$ and $-0.728 \mathrm{~km}$, respectively, in the $\mathrm{x}$ and $\mathrm{y}$ directions of the Moon-fixed coordinate system. The deviation was mainly along the direction of the Earth-Moon. It was estimated that the lunar crust on the far side was $10 \mathrm{~km}$ thicker than on the near side, which might be a major cause of the deviation of the lunar COM and COF towards the Earth's direction [18].

\section{DEM Accuracy Assessment}

In this section, we compared the accuracy of different DEMs using the positions of known control points on the lunar surface. The spectral analysis was also adopted to evaluate the DEM accuracy.

4.1. Accuracy Assessment with LLRR and ALSEP. As shown in Figure 5, LLRR (Lunar Laser Ranging Reflector) was a series of laser reflectors placed by Lunakhod, Apollo 11, Apollo 14, and Apollo 15 on the near side of the Moon. ALSEP (Apollo Lunar Surface Experiments Package) was an experimental setup equipped with radio transponders placed during Apollo missions 12,14,15,16, and 17. The coordinate accuracy of LLRR and ALSEP was less than $3 \mathrm{~m}$ and $30 \mathrm{~m}$, respectively $[19,20]$. We could interpolate the topography model to calculate the elevation of the coordinates of LLRR and ALSEP landmarks and compared them with the elevation values of LLRR and ALSEP themselves to measure the accuracy of the lunar DEM. The results were listed in Table 4.

In Table 4, the difference between original LAM and LAM-2B-SHAO was from the orbit improvement, which had an improvement from $147.7 \mathrm{~m}$ to $127.3 \mathrm{~m}$. The accuracy improvement of the LAM-2B-SHAO-cali DEM when compared with LAM-2B-SHAO mainly came from the correction

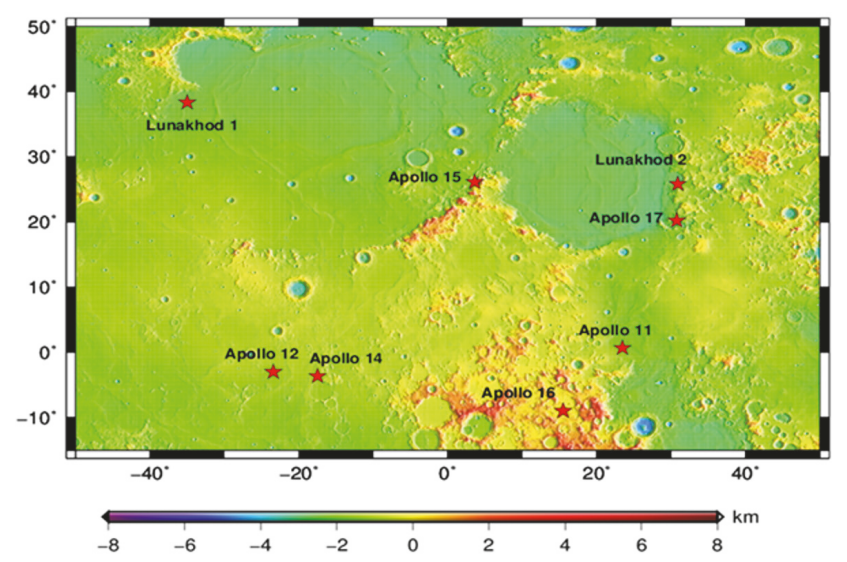

FIGURE 5: Distribution of LLRR and ALSEP on the lunar surface.

of $\sim 139 \mathrm{~m}$ bias. The improvement affected elevation directly, and the elevation accuracy of LLRR and ALSEP sites was improved obviously from $127.3 \mathrm{~m}$ to $48.7 \mathrm{~m}$.

For comparison, the result of LOLA was also showed in Table 4, and we could see that the accuracy of LOLA derived DEM is better than that of LAM data, not only for its higher precision of altimeter data but also for its higher space resolution.

4.2. Spectral Analysis. The variance $\sigma_{l}$ of various orders of the spherical harmonic model could reflect the intensity of the spatial spectrum signal of the lunar topographic model:

$$
\sigma_{l}=\sqrt{\frac{\sum_{m=0}^{l}\left(\bar{C}_{l m}^{2}+\bar{S}_{l m}^{2}\right)}{2 l+1}}
$$




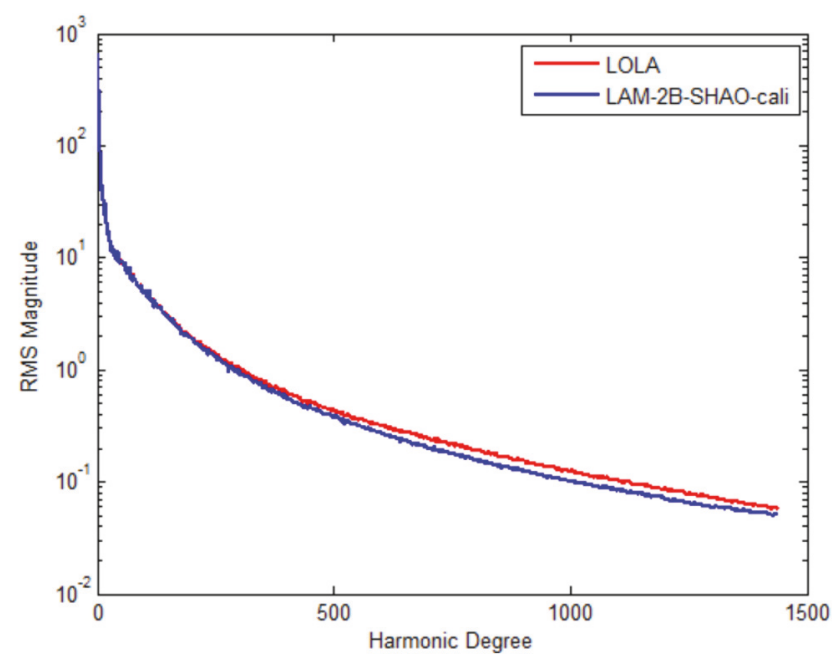

FIGURE 6: Power spectrum of CE-1 DEM and LRO DEM.

It could be seen from Figure 6 that CE-1 DEM and LRO DEM were highly consistent at a lower order. After the 200th order, the power spectrum of the LOLA model was higher than the LAM models. This meant that the LOLA DEM had a higher spatial resolution at small scales and could provide more topography information.

\section{Conclusion}

In this paper, an empirical calibration method was proposed to calibrate the systematic errors in CE-1 LAM data. A new lunar DEM was derived using the calibrated LAM data. The DEM accuracy was evaluated by the comparison with LLRR and ALSEP reference coordinates. The results showed that the DEM accuracy was improved after the correction of the systematic errors in LAM data.

The result suggested that the constant bias of LAM was about - $139.52 \mathrm{~m}$. A lunar DEM grid model with the spatial resolution of $0.0625^{\circ} \times 0.0625^{\circ}$ was obtained based on the calibrated LAM data. After calibration, the DEM accuracy was improved from about $127.3 \mathrm{~m}$ to about $48.7 \mathrm{~m}$, compared with the coordinates of LLRR and ALSEP landmarks on the lunar surface.

\section{Data Availability}

The data used to support the findings of this study are available from the corresponding author upon request.

\section{Conflicts of Interest}

The authors declare that they have no conflicts of interest.

\section{Acknowledgments}

This work was supported by the National Natural Science Foundation of China (Grants nos. 11473056 and 11403076), the Science and Technology Commission of Shanghai (Grant no. 12DZ2273300), the Planetary Sciences Laboratory of Chinese Academy of Sciences, the Lunar Exploration Project of China, and the Key Laboratory of Space Object Measurement. The authors also thank C.K. Shum (The Ohio State University) and H.S. Fok (Wuhan University) for their support.

\section{References}

[1] N. J. Potts, A. L. Gullikson, N. M. Curran et al., "Robotic traverse and sample return strategies for a lunar farside mission to the Schrödinger basin," Advances in Space Research, vol. 55, no. 4, pp. 1241-1254, 2015.

[2] H. Araki, S. Tazawa, H. Noda et al., "Lunar global shape and polar topography derived from Kaguya-LALT laser altimetry," Science, vol. 323, no. 5916, pp. 897-900, 2009.

[3] Z. Y. Ouyang, "Science Result of Chang'e-1 Lunar Orbiter and Mission Goals of Chang'e-2," Spacecraft Engineering, vol. 19, no. 5, pp. 1-6, 2010.

[4] J. Yan, J. Ping, F. Li et al., "Chang'E-1 precision orbit determination and lunar gravity field solution," Advances in Space Research, vol. 46, no. 1, pp. 50-57, 2010.

[5] J. Yan, S. Goossens, K. Matsumoto et al., "CEGM02: An improved lunar gravity model using Chang'E-1 orbital tracking data," Planetary and Space Science, vol. 62, no. 1, pp. 1-9, 2012.

[6] J. Nath Goswami, "Chandrayaan-1: India's first planetary science mission," Current Science, vol. 96, no. 4, 2008.

[7] R. Vondrak, J. Keller, G. Chin, and J. Garvin, "Lunar reconnaissance orbiter (LRO): Observations for lunar exploration and science," Space Science Reviews, vol. 150, no. 1-4, pp. 7-22, 2010.

[8] K. Di, W. Hu, Y. Liu, and M. Peng, "Co-registration of Chang'E1 stereo images and laser altimeter data with crossover adjustment and image sensor model refinement," Advances in Space Research, vol. 50, no. 12, pp. 1615-1628, 2012.

[9] S. Zhao, X. Ran, J. Fu, and Q. Guo, "Combined adjustment of CE-1 stereo camera image and laser altimeter data," Cehui Xuebao/Acta Geodaetica et Cartographica Sinica, vol. 43, no. 12, pp. 1224-1229, 2014.

[10] M. K. Barker, E. Mazarico, G. A. Neumann, M. T. Zuber, J. Haruyama, and D. E. Smith, "A new lunar digital elevation model from the Lunar Orbiter Laser Altimeter and SELENE Terrain Camera," Icarus, vol. 273, pp. 346-355, 2016.

[11] J. Ping S, Q. Huang, J. Yan G et al., "Lunar topographic model CLTM-s01 from ChangE-1 laser altimeter," Science in China Series G: Physics Mechanics and Astronomy, vol. 38, no. 11, pp. 1601-1612, 2008.

[12] C. Li L, X. Ren, J. Liu et al., "Laser Altimetry Data of Chang'E1 and the Global Lunar DEM Model," Science China: Earth Science, vol. 3, pp. 281-293, 2010.

[13] Z. Cai C, Y. Liang, J. Li et al., "Digital Elevation Model of the Moon from the ChangE-1 Laser Altimeter," Progress in Geophys (in Chinese), vol. 25, no. 4, pp. 1153-1160, 2010.

[14] W. Hu, K. Di, Z. Liu, and J. Ping, "A new lunar global DEM derived from chang'E-1 laser altimeter data based on crossover adjustment with local topographic constraint," Planetary and Space Science, vol. 87, pp. 173-182, 2013.

[15] D. E. Smith, M. T. Zuber, G. A. Neumann et al., "Initial observations from the Lunar Orbiter Laser Altimeter," Geophysical Research Letters, vol. 37, article L18204, 2010. 
[16] J. Ping, X. Su, J. Liu, R. Shu, and J. Yan, "External calibration for laser altimetry mission in Chang'E-1," Scientia Sinica Physica, Mechanica \& Astronomica, vol. 43, no. 11, pp. 1438-1447, 2013.

[17] S. Chang, Y. Huang, P. Li, X. Hu, and M. Fan, "The use of laser altimetry data in Chang'E-1 precision orbit determination," Research in Astronomy and Astrophysics, vol. 16, no. 9, pp. 83-90, 2016.

[18] D. Smith E, M. Zuber T, G. Neumann A et al., "Summary of the results from the lunar orbiter laser altimeter after seven years in lunar orbit," Icarus, vol. 283, pp. 70-91, 2016.

[19] M. E. Davies and T. R. Colvin, "Lunar coordinates in the regions of the Apollo landers," Journal of Geophysical Research: Planets, vol. 105, no. 8, pp. 20277-20280, 2000.

[20] H. S. Fok, C. K. Shum, Y. Yi et al., "Accuracy assessment of lunar topography models," Earth, Planets and Space, vol. 63, no. 1, pp. 15-23, 2011. 

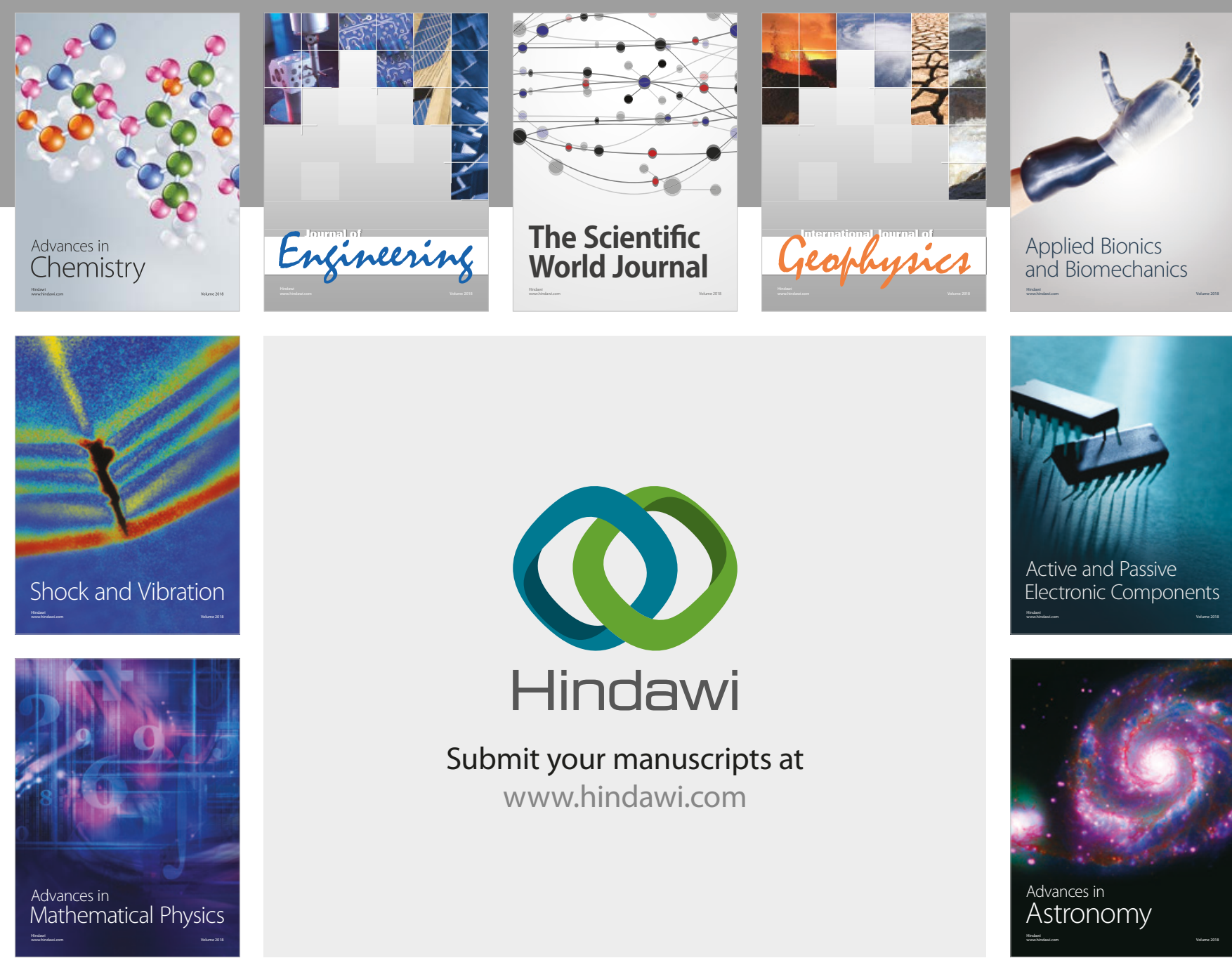

Submit your manuscripts at

www.hindawi.com

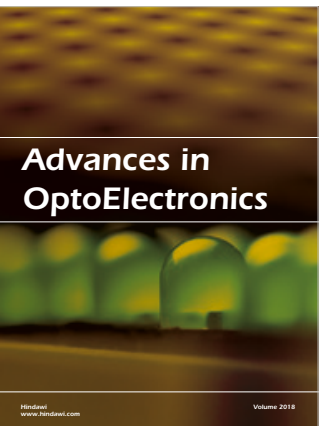

\section{Rotcting Machinery}
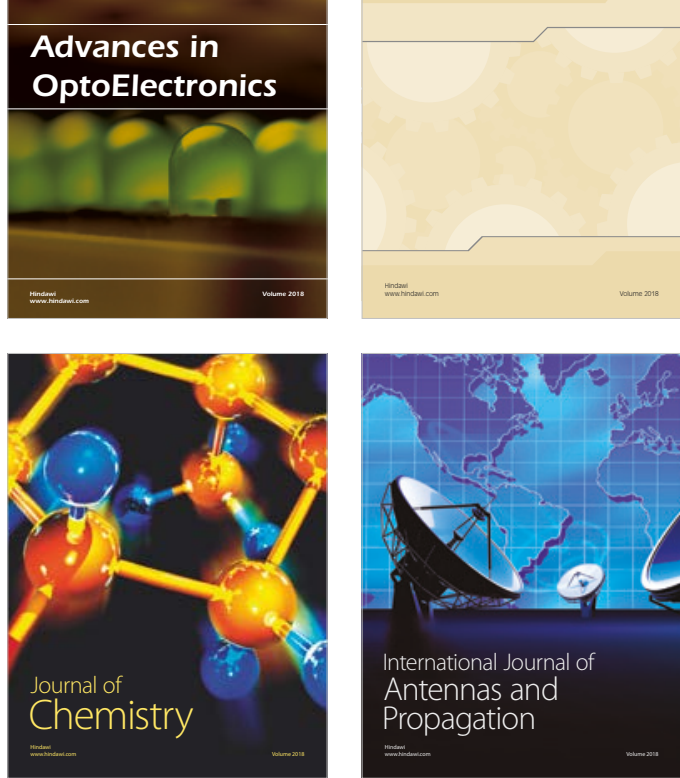

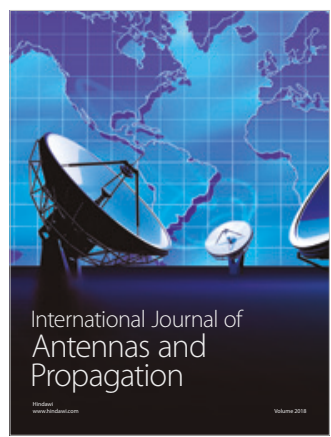

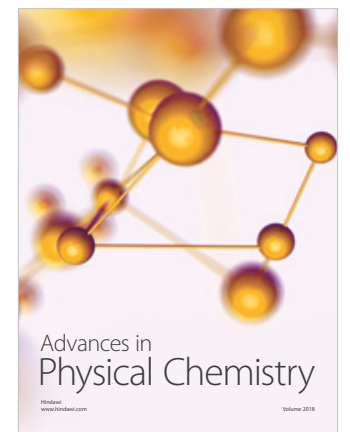

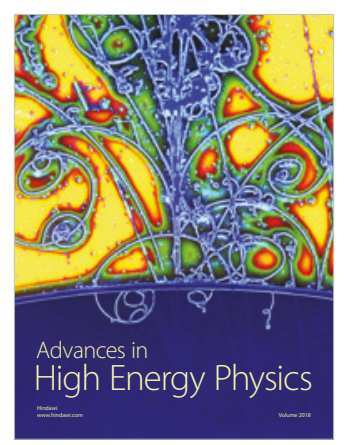

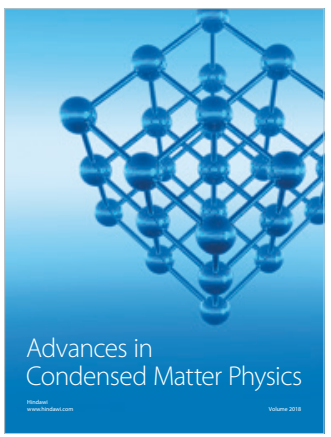

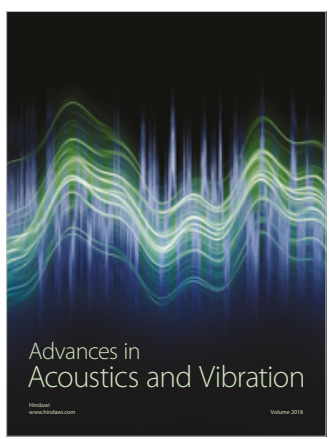

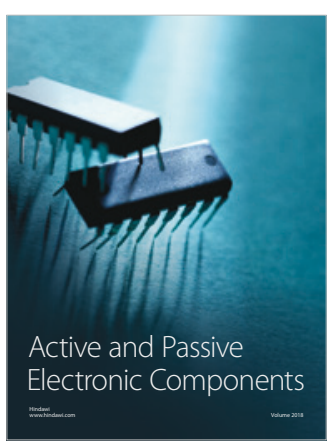
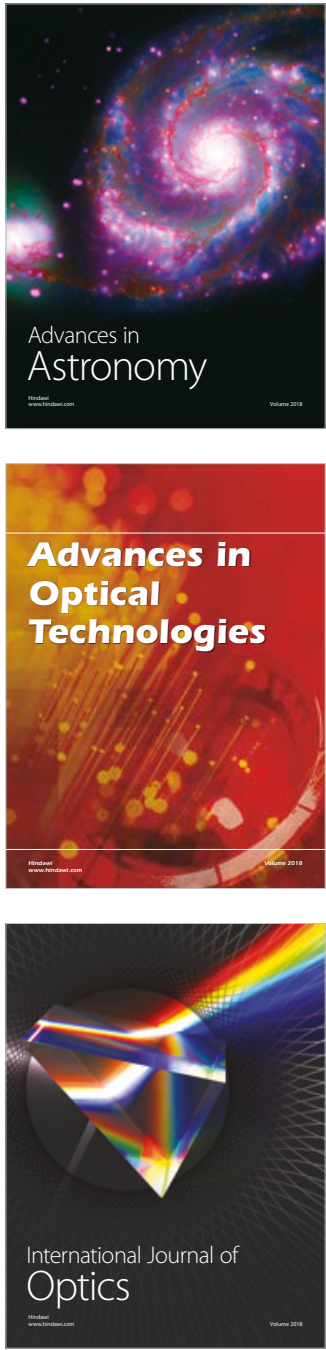